JOURNAL OF THE

AMERICAN MATHEMATICAL SOCIETY

Volume 22, Number 1, January 2009, Pages 287-307

S 0894-0347(08)00613-9

Article electronically published on July 17, 2008

\title{
MANIFOLDS WITH 1/4-PINCHED CURVATURE ARE SPACE FORMS
}

\author{
SIMON BRENDLE AND RICHARD SCHOEN
}

\section{INTRODUCTION}

One of the basic problems of Riemannian geometry is the classification of manifolds of positive sectional curvature. The known examples include the spherical space forms which carry constant curvature metrics and the rank 1 symmetric spaces whose canonical metrics have sectional curvatures at each point varying between 1 and 4. In 1951, H.E. Rauch [26] introduced the notion of curvature pinching for Riemannian manifolds and posed the question of whether a compact, simply connected manifold $M$ whose sectional curvatures all lie in the interval $(1,4]$ is necessarily homeomorphic to the sphere $S^{n}$. This was proven by M. Berger [2] and W. Klingenberg [18] around 1960 using comparison techniques. However, this theorem leaves open the question of whether $M$ is diffeomorphic to $S^{n}$. This conjecture is known as the Differentiable Sphere Theorem, and the purpose of this paper is to prove this and a more general result which we describe.

We will say that a manifold $M$ has pointwise $1 / 4$-pinched sectional curvatures if $M$ has positive sectional curvature and for every point $p \in M$ the ratio of the maximum to the minimum sectional curvature at that point is less than 4 . In other words, for every pair of two-planes $\pi_{1}, \pi_{2} \subset T_{p} M$ we have $0<K\left(\pi_{1}\right)<4 K\left(\pi_{2}\right)$. Our main result is the following:

Theorem 1. Let $M$ be a compact Riemannian manifold of dimension $n \geq 4$ with pointwise 1/4-pinched sectional curvatures. Then $M$ admits a metric of constant curvature and therefore is diffeomorphic to a spherical space form.

The techniques in this paper can be extended to give a classification of manifolds with weakly 1/4-pinched sectional curvatures. We refer to [4] for details.

Since our method of proof gives a canonical deformation from the 1/4-pinched metric to a constant curvature metric, we can also prove the following equivariant version.

Theorem 2. Let $M$ be a compact, simply connected Riemannian manifold of dimension $n \geq 4$ with pointwise 1/4-pinched sectional curvatures. Assume that $G$ is a compact Lie group and $\rho$ is a group homomorphism from $G$ into the isometry

Received by the editors May 7, 2007.

2000 Mathematics Subject Classification. Primary 53C20; Secondary 53C44.

Key words and phrases. Ricci flow, curvature pinching, sphere theorem.

The first author was partially supported by a Sloan Foundation Fellowship and by NSF grant DMS-0605223.

The second author was partially supported by NSF grant DMS-0604960.

(C)2008 American Mathematical Society 
group of $M$. Then there exists a group homomorphism $\sigma$ from $G$ into $O(n+1)$ and a diffeomorphism $F$ from $M$ to $S^{n}$ which is equivariant; i.e. $F \circ \rho(g)=\sigma(g) \circ F$ for all $g \in G$.

Notice that Theorem 1 and Theorem 2 do not assume any global pinching condition. A manifold is said to be globally $\delta$-pinched if all sectional curvatures at all points of $M$ lie in the interval $\left(1, \frac{1}{\delta}\right]$. The Differentiable Sphere Theorem under global $\delta$-pinching assumptions was obtained in 1966 by D. Gromoll [8] and E. Calabi with a constant $\delta=\delta(n)$ converging to 1 as $n \rightarrow \infty$. In 1971, M. Sugimoto, K. Shiohama, and H. Karcher [29] proved the Differentiable Sphere Theorem with a pinching constant independent of $n(\delta=0.87)$. The pinching constant was subsequently improved by E. Ruh [27] $(\delta=0.80)$ and by K. Grove, H. Karcher, and E. Ruh 10] $(\delta=0.76)$. Ruh 28 proved the Differentiable Sphere Theorem under a pointwise pinching condition, with a pinching constant converging to 1 as $n \rightarrow \infty$.

The equivariant sphere theorem was first proven for globally $\delta$-pinched manifolds by K. Grove, H. Karcher, and E. Ruh [9, 10] with a pinching constant $\delta$ independent of $n(\delta=0.98)$. The pinching constant was later improved by H. Im Hof and E. Ruh [16].

In 1982, R. Hamilton 11 introduced a fundamental new tool to this problem. Given a compact Riemannian manifold $\left(M, g_{0}\right)$, Hamilton evolved the Riemannian metric by the equation

$$
\frac{\partial}{\partial t} g(t)=-2 \operatorname{Ric}_{g(t)}
$$

with initial condition $g(0)=g_{0}$. This equation is known as the Ricci flow. Hamilton also defined a normalized version of the Ricci flow. The normalized Ricci flow is defined by

$$
\frac{\partial}{\partial t} g(t)=-2 \operatorname{Ric}_{g(t)}+\frac{2}{n} r_{g(t)} g(t)
$$

where $r_{g(t)}$ denotes the mean value of the scalar curvature of $g(t)$. Note that the volume of $M$ is constant under the normalized flow.

Using this method, Hamilton 11 proved that every three-manifold with positive Ricci curvature admits a constant curvature metric. In a subsequent paper, Hamilton [12] laid the general framework for the application of Ricci flow to Riemannian geometry and showed that four-manifolds with positive curvature operator are space forms. In 1991, H. Chen [6] extended this result to four-manifolds with 2-positive curvature operator, which implies Theorem 1 for $n=4$. More recently, B. Andrews and H. Nguyen [1] proved that four-manifolds with 1/4-pinched flag curvature are space forms. In higher dimensions, the Ricci flow was used by G. Huisken 15 to show that sufficiently pinched manifolds are space forms (see also [19, 23]).

C. Böhm and B. Wilking [3] used the Ricci flow to prove that manifolds with 2-positive curvature operator are space forms. Most importantly, their work introduces new methods for deforming invariant sets and constructing pinching sets for the ODE on the space of curvature-type tensors arising from the evolution of the curvature. The curvature ODE was introduced by R. Hamilton [12] and had been exploited effectively in dimensions 3 and 4 .

In 1988, M. Micallef and J.D. Moore [20] introduced minimal surface techniques into this problem and proved the topological sphere theorem for pointwise 1/4pinched manifolds using variational theory for the energy functional on maps from $S^{2}$ to $M$. Another important contribution of their paper was that they introduced 
a new curvature condition, positive isotropic curvature. This condition arose from consideration of the second variation of energy for maps of surfaces into $M$. The condition says that for every orthonormal four-frame $\left\{e_{1}, e_{2}, e_{3}, e_{4}\right\}$ we have the inequality

$$
R_{1313}+R_{1414}+R_{2323}+R_{2424}-2 R_{1234}>0 .
$$

If we allow the weak inequality, then we say that $M$ has nonnegative isotropic curvature. Micallef and Moore proved that a compact, simply connected manifold with positive isotropic curvature is homeomorphic to $S^{n}$. Moreover, they observed that pointwise 1/4-pinching implies positive isotropic curvature.

In dimension 4 , it was shown by R. Hamilton 14 that positive isotropic curvature is preserved by the Ricci flow. The Ricci flow on four-manifolds with positive isotropic curvature will, in general, develop singularities. Hamilton established pointwise estimates for the curvature tensor of the evolving metric and used them to give a precise description of the singularities in this situation [13, 14. In order to extend the flow beyond singularities, Hamilton introduced the notion of Ricci flow with surgeries (see also [5, 24], 25]).

In Section 2 we prove that positive isotropic curvature is preserved by the Ricci flow in all dimensions. By the maximum principle (cf. 12, Theorem 4.3), it suffices to show that positive isotropic curvature is preserved by the Hamilton ODE. We were not able to show that all isotropic curvatures improve under the ODE. Instead, we prove that the minimum isotropic curvature increases under the ODE, which is sufficient for our purposes. This is a very intricate calculation which exploits special identities and inequalities for the curvature tensor $R$ arising from the first and second variations applied to a set of four orthonormal vectors which minimize the isotropic curvature. After this paper was written, we learned that H. Nguyen 22. has independently proved that positive isotropic curvature is preserved under the Ricci flow.

Even knowing that positive isotropic curvature is preserved, it seems to be a difficult analytic problem to give a complete analysis of solutions to the Ricci flow satisfying that condition. A combination of results of M. Micallef and M. Wang [21] and recent results of A. Fraser [7] on nonsimply connected manifolds with positive isotropic curvature suggest that Hamilton's four-dimensional flow with surgeries may hold in all dimensions. We do not treat this question here. Instead, we establish a convergence result for the Ricci flow in dimension $n \geq 4$ under a curvature condition which is substantially stronger than positive isotropic curvature, but includes the pointwise 1/4-pinched manifolds.

Given a curvature tensor $R$ thought of as a four-tensor on $\mathbb{R}^{n}$, we define $\tilde{R}$ to be the extension of $R$ as a curvature tensor on $\mathbb{R}^{n} \times \mathbb{R}$ which is zero in the additional direction. Thus $\tilde{R}$ is the curvature tensor one obtains for the manifold $M \times \mathbb{R}$. The condition that $\tilde{R}$ has positive isotropic curvature is preserved by the Ricci flow and is a much stronger condition than positive isotropic curvature itself. If $\tilde{R}$ has positive isotropic curvature, then $R$ has 2-positive flag curvature in the sense that

$$
R_{1212}+R_{1313}>0
$$

for all orthonormal three-frames $\left\{e_{1}, e_{2}, e_{3}\right\}$. In particular, this condition implies that $R$ has positive Ricci curvature. Continuing in this vein, we define $\hat{R}$ to be the curvature tensor on $\mathbb{R}^{n} \times \mathbb{R}^{2}$ obtained by extending $R$ to be zero in the two additional directions. Thus $\hat{R}$ is the curvature tensor of $M \times \mathbb{R}^{2}$. The condition 
that $\hat{R}$ has nonnegative isotropic curvature is, again, preserved by the Ricci flow. Moreover, this condition implies that $R$ has nonnegative sectional curvature. Note that $\hat{R}$ cannot have positive isotropic curvature due to the two flat directions.

This construction provides us with a convex cone in the space of algebraic curvature operators which is invariant under the Hamilton ODE, is contained in the cone of curvature operators with nonnegative sectional curvature, and contains all nonnegative curvature operators. We may then directly apply results of Böhm and Wilking 3 to obtain suitable pinching sets for the ODE. Convergence of the normalized Ricci flow to a constant curvature metric then follows from work of Hamilton [12] (see also [3]). This material is discussed in detail in Section 3.

Finally, in Section 4 we give a necessary and sufficient condition for $\hat{R}$ to have nonnegative isotropic curvature. This yields the following result:

Theorem 3. Let $\left(M, g_{0}\right)$ be a compact Riemannian manifold of dimension $n \geq 4$. Assume that

$$
R_{1313}+\lambda^{2} R_{1414}+\mu^{2} R_{2323}+\lambda^{2} \mu^{2} R_{2424}-2 \lambda \mu R_{1234}>0
$$

for all orthonormal four-frames $\left\{e_{1}, e_{2}, e_{3}, e_{4}\right\}$ and all $\lambda, \mu \in[-1,1]$. Then the normalized Ricci flow with initial metric $g_{0}$ exists for all time and converges to a constant curvature metric as $t \rightarrow \infty$.

It follows from Berger's inequality that every manifold with pointwise 1/4pinched sectional curvatures satisfies the curvature condition in Theorem 3. Hence, Theorem 1 and Theorem 2 are immediate consequences of Theorem 3 ,

\section{Positive isotropic curvature is preserved by the Ricci flow}

In this section, we will prove that positive isotropic curvature is preserved by the Ricci flow. By work of R. Hamilton [12, it suffices to show that positive isotropic curvature is preserved by the ODE $\frac{d}{d t} R=Q(R)$, where $Q(R)$ is defined by

$$
Q(R)_{i j k l}=R_{i j p q} R_{k l p q}+2 R_{i p k q} R_{j p l q}-2 R_{i p l q} R_{j p k q} .
$$

To that end, we assume that $R$ is a curvature tensor with nonnegative isotropic curvature. Moreover, suppose that $\left\{e_{1}, e_{2}, e_{3}, e_{4}\right\}$ is an orthonormal four-frame satisfying

$$
R_{1313}+R_{1414}+R_{2323}+R_{2424}-2 R_{1234}=0 .
$$

We will show that

$$
Q(R)_{1313}+Q(R)_{1414}+Q(R)_{2323}+Q(R)_{2424}-2 Q(R)_{1234} \geq 0 .
$$

The following observation will be useful: if $\left\{e_{1}, e_{2}, e_{3}, e_{4}\right\}$ is an orthonormal fourframe satisfying (11), then the four-frames $\left\{e_{2},-e_{1}, e_{3}, e_{4}\right\},\left\{e_{2},-e_{1}, e_{4},-e_{3}\right\}$, and $\left\{e_{3}, e_{4}, e_{1}, e_{2}\right\}$ also satisfy (11). Hence, any statement that we can prove for the frame $\left\{e_{1}, e_{2}, e_{3}, e_{4}\right\}$ will also hold for the frames $\left\{e_{2},-e_{1}, e_{3}, e_{4}\right\},\left\{e_{2},-e_{1}, e_{4},-e_{3}\right\}$, and $\left\{e_{3}, e_{4}, e_{1}, e_{2}\right\}$.

Using the first Bianchi identity, we obtain

$$
\begin{aligned}
Q(R)_{1234} & =R_{12 p q} R_{34 p q}+2 R_{1 p 3 q} R_{2 p 4 q}-2 R_{1 p 4 q} R_{2 p 3 q} \\
& =R_{12 p q} R_{34 p q}+R_{13 p q} R_{24 p q}-R_{14 p q} R_{23 p q} \\
& +2 R_{1 p 3 q} R_{4 p 2 q}-2 R_{1 p 4 q} R_{3 p 2 q} .
\end{aligned}
$$


This implies

$$
\begin{aligned}
& Q(R)_{1313}+Q(R)_{1414}+Q(R)_{2323}+Q(R)_{2424}-2 Q(R)_{1234} \\
& =R_{13 p q} R_{13 p q}+2 R_{1 p 1 q} R_{3 p 3 q}-2 R_{1 p 3 q} R_{3 p 1 q} \\
& +R_{14 p q} R_{14 p q}+2 R_{1 p 1 q} R_{4 p 4 q}-2 R_{1 p 4 q} R_{4 p 1 q} \\
& +R_{23 p q} R_{23 p q}+2 R_{2 p 2 q} R_{3 p 3 q}-2 R_{2 p 3 q} R_{3 p 2 q} \\
& +R_{24 p q} R_{24 p q}+2 R_{2 p 2 q} R_{4 p 4 q}-2 R_{2 p 4 q} R_{4 p 2 q} \\
& -2 R_{12 p q} R_{34 p q}-2 R_{13 p q} R_{24 p q}+2 R_{14 p q} R_{23 p q} \\
& -4 R_{1 p 3 q} R_{4 p 2 q}+4 R_{1 p 4 q} R_{3 p 2 q}
\end{aligned}
$$

Rearranging terms yields

$$
\begin{aligned}
& Q(R)_{1313}+Q(R)_{1414}+Q(R)_{2323}+Q(R)_{2424}-2 Q(R)_{1234} \\
& =\left(R_{13 p q}-R_{24 p q}\right)\left(R_{13 p q}-R_{24 p q}\right)+\left(R_{14 p q}+R_{23 p q}\right)\left(R_{14 p q}+R_{23 p q}\right) \\
& +2\left(R_{1 p 1 q}+R_{2 p 2 q}\right)\left(R_{3 p 3 q}+R_{4 p 4 q}\right)-2 R_{12 p q} R_{34 p q} \\
& -2\left(R_{1 p 3 q}+R_{2 p 4 q}\right)\left(R_{3 p 1 q}+R_{4 p 2 q}\right)-2\left(R_{1 p 4 q}-R_{2 p 3 q}\right)\left(R_{4 p 1 q}-R_{3 p 2 q}\right) .
\end{aligned}
$$

The first two terms on the right are clearly nonnegative.

Lemma 4. We have

$$
R_{1213}+R_{1242}+R_{3413}+R_{3442}=R_{1214}+R_{1223}+R_{3414}+R_{3423}=0 .
$$

Proof. Consider the frame $\left\{e_{1}, \cos s e_{2}-\sin s e_{3}, \sin s e_{2}+\cos s e_{3}, e_{4}\right\}$. Since $R$ has nonnegative isotropic curvature, the function

$$
\begin{gathered}
s \mapsto \cos ^{2} s\left(R_{1313}+R_{2424}-2 R_{1234}\right)+\sin ^{2} s\left(R_{1212}+R_{3434}+2 R_{1324}\right) \\
+R_{1414}+R_{2323}+2 \cos s \sin s\left(R_{1213}-R_{2434}-R_{1224}+R_{1334}\right)
\end{gathered}
$$

is nonnegative and vanishes for $s=0$. This implies $R_{1213}-R_{2434}-R_{1224}+R_{1334}=0$. If we replace $\left\{e_{1}, e_{2}, e_{3}, e_{4}\right\}$ by $\left\{e_{2},-e_{1}, e_{3}, e_{4}\right\}$, we obtain $-R_{2123}+R_{1434}-R_{2114}+$ $R_{2334}=0$.

Proposition 5. We have

$$
\begin{aligned}
& \sum_{p, q=1}^{4}\left(R_{1 p 1 q}+R_{2 p 2 q}\right)\left(R_{3 p 3 q}+R_{4 p 4 q}\right)-\sum_{p, q=1}^{4} R_{12 p q} R_{34 p q} \\
& =\sum_{p, q=1}^{4}\left(R_{1 p 3 q}+R_{2 p 4 q}\right)\left(R_{3 p 1 q}+R_{4 p 2 q}\right) \\
& +\sum_{p, q=1}^{4}\left(R_{1 p 4 q}-R_{2 p 3 q}\right)\left(R_{4 p 1 q}-R_{3 p 2 q}\right) .
\end{aligned}
$$


Proof. Direct computation yields

$$
\begin{aligned}
\sum_{p, q=1}^{4}\left(R_{1 p 1 q}+R_{2 p 2 q}\right)\left(R_{3 p 3 q}+R_{4 p 4 q}\right)-\sum_{p, q=1}^{4} R_{12 p q} R_{34 p q} \\
-\sum_{p, q=1}^{4}\left(R_{1 p 3 q}+R_{2 p 4 q}\right)\left(R_{3 p 1 q}+R_{4 p 2 q}\right) \\
-\sum_{p, q=1}^{4}\left(R_{1 p 4 q}-R_{2 p 3 q}\right)\left(R_{4 p 1 q}-R_{3 p 2 q}\right) \\
=\left(R_{1212}+R_{3434}\right)\left(R_{1313}+R_{1414}+R_{2323}+R_{2424}-2 R_{1234}\right) \\
+2 R_{1234}\left(R_{1313}+R_{1414}+R_{2323}+R_{2424}+2 R_{1342}+2 R_{1423}\right) \\
-\left(R_{1213}+R_{1242}+R_{3413}+R_{3442}\right)^{2}-\left(R_{1214}+R_{1223}+R_{3414}+R_{3423}\right)^{2} \\
=\left(R_{1212}+R_{3434}+2 R_{1234}\right)\left(R_{1313}+R_{1414}+R_{2323}+R_{2424}-2 R_{1234}\right) \\
-\left(R_{1213}+R_{1242}+R_{3413}+R_{3442}\right)^{2}-\left(R_{1214}+R_{1223}+R_{3414}+R_{3423}\right)^{2} .
\end{aligned}
$$

The expression on the right is zero by Lemma 4

Lemma 6. We have

$$
R_{133 q}+R_{144 q}+R_{432 q}=R_{233 q}+R_{244 q}+R_{341 q}=0
$$

for all $5 \leq q \leq n$.

Proof. Consider the frame $\left\{\cos s e_{1}+\sin s e_{q}, e_{2}, e_{3}, e_{4}\right\}$. Since $R$ has nonnegative isotropic curvature, the function

$$
\begin{aligned}
s \mapsto & \cos ^{2} s\left(R_{1313}+R_{1414}\right)+\sin ^{2} s\left(R_{q 3 q 3}+R_{q 4 q 4}\right)+R_{2323}+R_{2424} \\
& +2 \cos s \sin s\left(R_{13 q 3}+R_{14 q 4}\right)-2 \cos s R_{1234}-2 \sin s R_{q 234}
\end{aligned}
$$

is nonnegative and vanishes for $s=0$. This implies $R_{13 q 3}+R_{14 q 4}-R_{q 234}=0$. If we replace $\left\{e_{1}, e_{2}, e_{3}, e_{4}\right\}$ by $\left\{e_{2},-e_{1}, e_{3}, e_{4}\right\}$, we obtain $R_{23 q 3}+R_{24 q 4}+R_{q 134}=0$.

Proposition 7. Fix $q$ such that $5 \leq q \leq n$. Then we have

$$
\begin{aligned}
& \sum_{p=1}^{4}\left(R_{1 p 1 q}+R_{2 p 2 q}\right)\left(R_{3 p 3 q}+R_{4 p 4 q}\right)-\sum_{p=1}^{4} R_{12 p q} R_{34 p q} \\
& =\sum_{p=1}^{4}\left(R_{1 p 3 q}+R_{2 p 4 q}\right)\left(R_{3 p 1 q}+R_{4 p 2 q}\right) \\
& +\sum_{p=1}^{4}\left(R_{1 p 4 q}-R_{2 p 3 q}\right)\left(R_{4 p 1 q}-R_{3 p 2 q}\right) .
\end{aligned}
$$


Proof. Using Lemma 6, we obtain

$$
\begin{aligned}
& \sum_{p=1}^{2}\left(R_{1 p 1 q}+R_{2 p 2 q}\right)\left(R_{3 p 3 q}+R_{4 p 4 q}\right)-\sum_{p=1}^{2} R_{12 p q} R_{34 p q} \\
& =R_{212 q}\left(R_{313 q}+R_{414 q}\right)+R_{121 q}\left(R_{323 q}+R_{424 q}\right) \\
& -R_{121 q} R_{341 q}-R_{122 q} R_{342 q} \\
& =R_{212 q}\left(R_{313 q}+R_{414 q}+R_{342 q}\right) \\
& +R_{121 q}\left(R_{323 q}+R_{424 q}-R_{341 q}\right) \\
& =0
\end{aligned}
$$

and

$$
\begin{aligned}
& \sum_{p=3}^{4}\left(R_{1 p 3 q}+R_{2 p 4 q}\right)\left(R_{3 p 1 q}+R_{4 p 2 q}\right) \\
& +\sum_{p=3}^{4}\left(R_{1 p 4 q}-R_{2 p 3 q}\right)\left(R_{4 p 1 q}-R_{3 p 2 q}\right) \\
& =\left(R_{133 q}+R_{234 q}\right) R_{432 q}+\left(R_{143 q}+R_{244 q}\right) R_{341 q} \\
& +\left(R_{134 q}-R_{233 q}\right) R_{431 q}-\left(R_{144 q}-R_{243 q}\right) R_{342 q} \\
& =\left(R_{133 q}+R_{234 q}+R_{144 q}-R_{243 q}\right) R_{432 q} \\
& +\left(R_{143 q}+R_{244 q}-R_{134 q}+R_{233 q}\right) R_{341 q} \\
& =\left(R_{133 q}+R_{144 q}+R_{432 q}\right) R_{432 q} \\
& +\left(R_{341 q}+R_{244 q}+R_{233 q}\right) R_{341 q} \\
& =0 .
\end{aligned}
$$

Replacing $\left\{e_{1}, e_{2}, e_{3}, e_{4}\right\}$ by $\left\{e_{3}, e_{4}, e_{1}, e_{2}\right\}$ yields

$$
\sum_{p=3}^{4}\left(R_{1 p 1 q}+R_{2 p 2 q}\right)\left(R_{3 p 3 q}+R_{4 p 4 q}\right)-\sum_{p=3}^{4} R_{12 p q} R_{34 p q}=0
$$

and

$$
\begin{aligned}
& \sum_{p=1}^{2}\left(R_{1 p 3 q}+R_{2 p 4 q}\right)\left(R_{3 p 1 q}+R_{4 p 2 q}\right) \\
& +\sum_{p=1}^{2}\left(R_{1 p 4 q}-R_{2 p 3 q}\right)\left(R_{4 p 1 q}-R_{3 p 2 q}\right)=0 .
\end{aligned}
$$

Putting these facts together, the assertion follows. 
Proposition 8. Assume that $w_{1}, w_{2}, w_{3}, w_{4}$ are orthogonal to $e_{1}, e_{2}, e_{3}, e_{4}$. Then the expression

$$
\begin{aligned}
& R\left(w_{1}, e_{3}, w_{1}, e_{3}\right)+R\left(w_{1}, e_{4}, w_{1}, e_{4}\right) \\
& +R\left(w_{2}, e_{3}, w_{2}, e_{3}\right)+R\left(w_{2}, e_{4}, w_{2}, e_{4}\right) \\
& +R\left(e_{1}, w_{3}, e_{1}, w_{3}\right)+R\left(e_{2}, w_{3}, e_{2}, w_{3}\right) \\
& +R\left(e_{1}, w_{4}, e_{1}, w_{4}\right)+R\left(e_{2}, w_{4}, e_{2}, w_{4}\right) \\
& -2\left[R\left(e_{3}, w_{1}, e_{1}, w_{3}\right)+R\left(e_{4}, w_{1}, e_{2}, w_{3}\right)\right] \\
& -2\left[R\left(e_{4}, w_{1}, e_{1}, w_{4}\right)-R\left(e_{3}, w_{1}, e_{2}, w_{4}\right)\right] \\
& +2\left[R\left(e_{4}, w_{2}, e_{1}, w_{3}\right)-R\left(e_{3}, w_{2}, e_{2}, w_{3}\right)\right] \\
& -2\left[R\left(e_{3}, w_{2}, e_{1}, w_{4}\right)+R\left(e_{4}, w_{2}, e_{2}, w_{4}\right)\right] \\
& -2 R\left(w_{1}, w_{2}, e_{3}, e_{4}\right)-2 R\left(e_{1}, e_{2}, w_{3}, w_{4}\right)
\end{aligned}
$$

is nonnegative.

Proof. For $i=1, \ldots, 4$, we denote by $v_{i}(s)$ the solution of the ODE

$$
v_{i}^{\prime}(s)=\sum_{j=1}^{4}\left(\left\langle v_{i}(s), e_{j}\right\rangle w_{j}-\left\langle v_{i}(s), w_{j}\right\rangle e_{j}\right)
$$

with initial condition $v_{i}(0)=e_{i}$. Clearly, $\left\{v_{1}(s), v_{2}(s), v_{3}(s), v_{4}(s)\right\}$ is an orthonormal four-frame. Moreover, $v_{i}^{\prime}(0)=w_{i}$ and $v_{i}^{\prime \prime}(0)=-\sum_{j=1}^{4}\left\langle w_{i}, w_{j}\right\rangle e_{j}$. Since $R$ has nonnegative isotropic curvature, the function

$$
\begin{aligned}
s \mapsto & R\left(v_{1}(s), v_{3}(s), v_{1}(s), v_{3}(s)\right)+R\left(v_{1}(s), v_{4}(s), v_{1}(s), v_{4}(s)\right) \\
& +R\left(v_{2}(s), v_{3}(s), v_{2}(s), v_{3}(s)\right)+R\left(v_{2}(s), v_{4}(s), v_{2}(s), v_{4}(s)\right) \\
& -2 R\left(v_{1}(s), v_{2}(s), v_{3}(s), v_{4}(s)\right)
\end{aligned}
$$

is nonnegative and vanishes for $s=0$. Therefore, the second derivative of this function at $s=0$ is nonnegative. This implies

$$
0 \leq J^{(1)}+J^{(2)}+J^{(3)}+J^{(4)}-J^{(5)}
$$

where

$$
\begin{aligned}
J^{(1)} & =\left.\frac{1}{2} \frac{d^{2}}{d s^{2}} R\left(v_{1}(s), v_{3}(s), v_{1}(s), v_{3}(s)\right)\right|_{s=0} \\
& =R\left(w_{1}, e_{3}, w_{1}, e_{3}\right)+R\left(e_{1}, w_{3}, e_{1}, w_{3}\right) \\
& +2 R\left(e_{1}, e_{3}, w_{1}, w_{3}\right)+2 R\left(e_{1}, w_{3}, w_{1}, e_{3}\right) \\
& -\left(\left|w_{1}\right|^{2}+\left|w_{3}\right|^{2}\right) R\left(e_{1}, e_{3}, e_{1}, e_{3}\right) \\
& -\left\langle w_{1}, w_{2}\right\rangle R\left(e_{1}, e_{3}, e_{2}, e_{3}\right)-\left\langle w_{1}, w_{4}\right\rangle R\left(e_{1}, e_{3}, e_{4}, e_{3}\right) \\
& -\left\langle w_{3}, w_{2}\right\rangle R\left(e_{1}, e_{3}, e_{1}, e_{2}\right)-\left\langle w_{3}, w_{4}\right\rangle R\left(e_{1}, e_{3}, e_{1}, e_{4}\right),
\end{aligned}
$$




$$
\begin{aligned}
J^{(2)} & =\left.\frac{1}{2} \frac{d^{2}}{d s^{2}} R\left(v_{1}(s), v_{4}(s), v_{1}(s), v_{4}(s)\right)\right|_{s=0} \\
& =R\left(w_{1}, e_{4}, w_{1}, e_{4}\right)+R\left(e_{1}, w_{4}, e_{1}, w_{4}\right) \\
& +2 R\left(e_{1}, e_{4}, w_{1}, w_{4}\right)+2 R\left(e_{1}, w_{4}, w_{1}, e_{4}\right) \\
& -\left(\left|w_{1}\right|^{2}+\left|w_{4}\right|^{2}\right) R\left(e_{1}, e_{4}, e_{1}, e_{4}\right) \\
& -\left\langle w_{1}, w_{2}\right\rangle R\left(e_{1}, e_{4}, e_{2}, e_{4}\right)-\left\langle w_{1}, w_{3}\right\rangle R\left(e_{1}, e_{4}, e_{3}, e_{4}\right) \\
& -\left\langle w_{4}, w_{2}\right\rangle R\left(e_{1}, e_{4}, e_{1}, e_{2}\right)-\left\langle w_{4}, w_{3}\right\rangle R\left(e_{1}, e_{4}, e_{1}, e_{3}\right), \\
& \\
J^{(3)} & =\left.\frac{1}{2} \frac{d^{2}}{d s^{2}} R\left(v_{2}(s), v_{3}(s), v_{2}(s), v_{3}(s)\right)\right|_{s=0} \\
& =R\left(w_{2}, e_{3}, w_{2}, e_{3}\right)+R\left(e_{2}, w_{3}, e_{2}, w_{3}\right) \\
& +2 R\left(e_{2}, e_{3}, w_{2}, w_{3}\right)+2 R\left(e_{2}, w_{3}, w_{2}, e_{3}\right) \\
& -\left(\left|w_{2}\right|^{2}+\left|w_{3}\right|^{2}\right) R\left(e_{2}, e_{3}, e_{2}, e_{3}\right) \\
& -\left\langle w_{2}, w_{1}\right\rangle R\left(e_{2}, e_{3}, e_{1}, e_{3}\right)-\left\langle w_{2}, w_{4}\right\rangle R\left(e_{2}, e_{3}, e_{4}, e_{3}\right) \\
& -\left\langle w_{3}, w_{1}\right\rangle R\left(e_{2}, e_{3}, e_{2}, e_{1}\right)-\left\langle w_{3}, w_{4}\right\rangle R\left(e_{2}, e_{3}, e_{2}, e_{4}\right), \\
& \\
& -\left\langle w_{4}, w_{1}\right\rangle R\left(e_{2}, e_{4}, e_{2}, e_{1}\right)-\left\langle w_{4}, w_{3}\right\rangle R\left(e_{2}, e_{4}, e_{2}, e_{3}\right), \\
& =2 R\left(e_{2}, e_{4}, w_{2}, w_{4}\right)+2 R\left(e_{2}, w_{4}, w_{2}, e_{4}\right) \\
J^{(4)} & \left.=\left.\frac{1}{2} \frac{d^{2}}{d s^{2}} R\left(v_{2}(s), v_{4}(s), v_{2}(s), v_{4}(s)\right)\right|_{s=0}\right) R\left(e_{2}, e_{4}, e_{2}, e_{4}\right) \\
& =R\left(w_{2}, e_{4}, w_{2}, e_{4}\right)+R\left(e_{2}, w_{4}, e_{2}, w_{4}\right) \\
& \\
& \\
&
\end{aligned}
$$

and

$$
\begin{aligned}
J^{(5)} & =\left.\frac{d^{2}}{d s^{2}} R\left(v_{1}(s), v_{2}(s), v_{3}(s), v_{4}(s)\right)\right|_{s=0} \\
& =2 R\left(w_{1}, w_{2}, e_{3}, e_{4}\right)+2 R\left(w_{1}, e_{2}, w_{3}, e_{4}\right)+2 R\left(w_{1}, e_{2}, e_{3}, w_{4}\right) \\
& +2 R\left(e_{1}, w_{2}, w_{3}, e_{4}\right)+2 R\left(e_{1}, w_{2}, e_{3}, w_{4}\right)+2 R\left(e_{1}, e_{2}, w_{3}, w_{4}\right) \\
& -\left(\left|w_{1}\right|^{2}+\left|w_{2}\right|^{2}+\left|w_{3}\right|^{2}+\left|w_{4}\right|^{2}\right) R\left(e_{1}, e_{2}, e_{3}, e_{4}\right) \\
& -\left\langle w_{1}, w_{3}\right\rangle R\left(e_{3}, e_{2}, e_{3}, e_{4}\right)-\left\langle w_{1}, w_{4}\right\rangle R\left(e_{4}, e_{2}, e_{3}, e_{4}\right) \\
& -\left\langle w_{2}, w_{3}\right\rangle R\left(e_{1}, e_{3}, e_{3}, e_{4}\right)-\left\langle w_{2}, w_{4}\right\rangle R\left(e_{1}, e_{4}, e_{3}, e_{4}\right) \\
& -\left\langle w_{3}, w_{1}\right\rangle R\left(e_{1}, e_{2}, e_{1}, e_{4}\right)-\left\langle w_{3}, w_{2}\right\rangle R\left(e_{1}, e_{2}, e_{2}, e_{4}\right) \\
& -\left\langle w_{4}, w_{1}\right\rangle R\left(e_{1}, e_{2}, e_{3}, e_{1}\right)-\left\langle w_{4}, w_{2}\right\rangle R\left(e_{1}, e_{2}, e_{3}, e_{2}\right) .
\end{aligned}
$$


Rearranging terms yields

$$
\begin{aligned}
0 & \leq R\left(w_{1}, e_{3}, w_{1}, e_{3}\right)+R\left(w_{1}, e_{4}, w_{1}, e_{4}\right) \\
& +R\left(w_{2}, e_{3}, w_{2}, e_{3}\right)+R\left(w_{2}, e_{4}, w_{2}, e_{4}\right) \\
& +R\left(e_{1}, w_{3}, e_{1}, w_{3}\right)+R\left(e_{2}, w_{3}, e_{2}, w_{3}\right) \\
& +R\left(e_{1}, w_{4}, e_{1}, w_{4}\right)+R\left(e_{2}, w_{4}, e_{2}, w_{4}\right) \\
& +2 R\left(e_{1}, e_{3}, w_{1}, w_{3}\right)+2 R\left(e_{1}, w_{3}, w_{1}, e_{3}\right)-2 R\left(w_{1}, e_{2}, w_{3}, e_{4}\right) \\
& +2 R\left(e_{1}, e_{4}, w_{1}, w_{4}\right)+2 R\left(e_{1}, w_{4}, w_{1}, e_{4}\right)-2 R\left(w_{1}, e_{2}, e_{3}, w_{4}\right) \\
& +2 R\left(e_{2}, e_{3}, w_{2}, w_{3}\right)+2 R\left(e_{2}, w_{3}, w_{2}, e_{3}\right)-2 R\left(e_{1}, w_{2}, w_{3}, e_{4}\right) \\
& +2 R\left(e_{2}, e_{4}, w_{2}, w_{4}\right)+2 R\left(e_{2}, w_{4}, w_{2}, e_{4}\right)-2 R\left(e_{1}, w_{2}, e_{3}, w_{4}\right) \\
& -2 R\left(w_{1}, w_{2}, e_{3}, e_{4}\right)-2 R\left(e_{1}, e_{2}, w_{3}, w_{4}\right) \\
& -\left|w_{1}\right|^{2}\left(R_{1313}+R_{1414}-R_{1234}\right)-\left|w_{2}\right|^{2}\left(R_{2323}+R_{2424}-R_{1234}\right) \\
& -\left|w_{3}\right|^{2}\left(R_{1313}+R_{2323}-R_{1234}\right)-\left|w_{4}\right|^{2}\left(R_{1414}+R_{2424}-R_{1234}\right) \\
& +\left(\left\langle w_{1}, w_{3}\right\rangle-\left\langle w_{2}, w_{4}\right\rangle\right)\left(R_{1214}-R_{1232}+R_{3234}-R_{1434}\right) \\
& -\left(\left\langle w_{1}, w_{4}\right\rangle+\left\langle w_{2}, w_{3}\right\rangle\right)\left(R_{1213}+R_{1242}+R_{3134}+R_{2434}\right) \\
& -2\left\langle w_{1}, w_{2}\right\rangle\left(R_{1323}+R_{1424}\right)-2\left\langle w_{3}, w_{4}\right\rangle\left(R_{1314}+R_{2324}\right) .
\end{aligned}
$$

We now replace the frame $\left\{e_{1}, e_{2}, e_{3}, e_{4}\right\}$ by $\left\{e_{2},-e_{1}, e_{4},-e_{3}\right\}$. This yields

$$
\begin{aligned}
0 & \leq R\left(w_{1}, e_{4}, w_{1}, e_{4}\right)+R\left(w_{1}, e_{3}, w_{1}, e_{3}\right) \\
& +R\left(w_{2}, e_{4}, w_{2}, e_{4}\right)+R\left(w_{2}, e_{3}, w_{2}, e_{3}\right) \\
& +R\left(e_{2}, w_{3}, e_{2}, w_{3}\right)+R\left(e_{1}, w_{3}, e_{1}, w_{3}\right) \\
& +R\left(e_{2}, w_{4}, e_{2}, w_{4}\right)+R\left(e_{1}, w_{4}, e_{1}, w_{4}\right) \\
& +2 R\left(e_{2}, e_{4}, w_{1}, w_{3}\right)+2 R\left(e_{2}, w_{3}, w_{1}, e_{4}\right)-2 R\left(w_{1}, e_{1}, w_{3}, e_{3}\right) \\
& -2 R\left(e_{2}, e_{3}, w_{1}, w_{4}\right)-2 R\left(e_{2}, w_{4}, w_{1}, e_{3}\right)+2 R\left(w_{1}, e_{1}, e_{4}, w_{4}\right) \\
& -2 R\left(e_{1}, e_{4}, w_{2}, w_{3}\right)-2 R\left(e_{1}, w_{3}, w_{2}, e_{4}\right)+2 R\left(e_{2}, w_{2}, w_{3}, e_{3}\right) \\
& +2 R\left(e_{1}, e_{3}, w_{2}, w_{4}\right)+2 R\left(e_{1}, w_{4}, w_{2}, e_{3}\right)-2 R\left(e_{2}, w_{2}, e_{4}, w_{4}\right) \\
& +2 R\left(w_{1}, w_{2}, e_{4}, e_{3}\right)+2 R\left(e_{2}, e_{1}, w_{3}, w_{4}\right) \\
& -\left|w_{1}\right|^{2}\left(R_{2424}+R_{2323}-R_{2143}\right)-\left|w_{2}\right|^{2}\left(R_{1414}+R_{1313}-R_{2143}\right) \\
& -\left|w_{3}\right|^{2}\left(R_{2424}+R_{1414}-R_{2143}\right)-\left|w_{4}\right|^{2}\left(R_{2323}+R_{1313}-R_{2143}\right) \\
& +\left(\left\langle w_{1}, w_{3}\right\rangle-\left\langle w_{2}, w_{4}\right\rangle\right)\left(R_{2123}-R_{2141}+R_{4143}-R_{2343}\right) \\
& +\left(\left\langle w_{1}, w_{4}\right\rangle+\left\langle w_{2}, w_{3}\right\rangle\right)\left(R_{2124}+R_{2131}+R_{4243}+R_{1343}\right) \\
& +2\left\langle w_{1}, w_{2}\right\rangle\left(R_{2414}+R_{2313}\right)+2\left\langle w_{3}, w_{4}\right\rangle\left(R_{2423}+R_{1413}\right) .
\end{aligned}
$$


In the next step, we take the arithmetic mean of both inequalitities. Using the identity $R_{1313}+R_{1414}+R_{2323}+R_{2424}-2 R_{1234}=0$, we obtain

$$
\begin{aligned}
& 0 \leq R\left(w_{1}, e_{3}, w_{1}, e_{3}\right)+R\left(w_{1}, e_{4}, w_{1}, e_{4}\right) \\
& +R\left(w_{2}, e_{3}, w_{2}, e_{3}\right)+R\left(w_{2}, e_{4}, w_{2}, e_{4}\right) \\
& +R\left(e_{1}, w_{3}, e_{1}, w_{3}\right)+R\left(e_{2}, w_{3}, e_{2}, w_{3}\right) \\
& +R\left(e_{1}, w_{4}, e_{1}, w_{4}\right)+R\left(e_{2}, w_{4}, e_{2}, w_{4}\right) \\
& +\left[R\left(e_{1}, e_{3}, w_{1}, w_{3}\right)+R\left(e_{1}, w_{3}, w_{1}, e_{3}\right)-R\left(w_{1}, e_{2}, w_{3}, e_{4}\right)\right. \\
& \left.+R\left(e_{2}, e_{4}, w_{1}, w_{3}\right)+R\left(e_{2}, w_{3}, w_{1}, e_{4}\right)-R\left(w_{1}, e_{1}, w_{3}, e_{3}\right)\right] \\
& +\left[R\left(e_{1}, e_{4}, w_{1}, w_{4}\right)+R\left(e_{1}, w_{4}, w_{1}, e_{4}\right)-R\left(w_{1}, e_{2}, e_{3}, w_{4}\right)\right. \\
& \left.-R\left(e_{2}, e_{3}, w_{1}, w_{4}\right)-R\left(e_{2}, w_{4}, w_{1}, e_{3}\right)+R\left(w_{1}, e_{1}, e_{4}, w_{4}\right)\right] \\
& +\left[R\left(e_{2}, e_{3}, w_{2}, w_{3}\right)+R\left(e_{2}, w_{3}, w_{2}, e_{3}\right)-R\left(e_{1}, w_{2}, w_{3}, e_{4}\right)\right. \\
& \left.-R\left(e_{1}, e_{4}, w_{2}, w_{3}\right)-R\left(e_{1}, w_{3}, w_{2}, e_{4}\right)+R\left(e_{2}, w_{2}, w_{3}, e_{3}\right)\right] \\
& +\left[R\left(e_{2}, e_{4}, w_{2}, w_{4}\right)+R\left(e_{2}, w_{4}, w_{2}, e_{4}\right)-R\left(e_{1}, w_{2}, e_{3}, w_{4}\right)\right. \\
& \left.+R\left(e_{1}, e_{3}, w_{2}, w_{4}\right)+R\left(e_{1}, w_{4}, w_{2}, e_{3}\right)-R\left(e_{2}, w_{2}, e_{4}, w_{4}\right)\right] \\
& -2 R\left(w_{1}, w_{2}, e_{3}, e_{4}\right)-2 R\left(e_{1}, e_{2}, w_{3}, w_{4}\right) \text {. }
\end{aligned}
$$

The assertion follows now from the first Bianchi identity.

Proposition 9. We have

$$
\begin{aligned}
& \sum_{p, q=5}^{n}\left(R_{1 p 1 q}+R_{2 p 2 q}\right)\left(R_{3 p 3 q}+R_{4 p 4 q}\right)-\sum_{p, q=5}^{n} R_{12 p q} R_{34 p q} \\
& \geq \sum_{p, q=5}^{n}\left(R_{1 p 3 q}+R_{2 p 4 q}\right)\left(R_{3 p 1 q}+R_{4 p 2 q}\right) \\
& +\sum_{p, q=5}^{n}\left(R_{1 p 4 q}-R_{2 p 3 q}\right)\left(R_{4 p 1 q}-R_{3 p 2 q}\right) .
\end{aligned}
$$

Proof. Consider the following $(n-4) \times(n-4)$ matrices:

$$
\begin{aligned}
a_{p q} & =R_{1 p 1 q}+R_{2 p 2 q}, & b_{p q} & =R_{3 p 3 q}+R_{4 p 4 q}, \\
c_{p q} & =R_{3 p 1 q}+R_{4 p 2 q}, & & d_{p q}=R_{4 p 1 q}-R_{3 p 2 q}, \\
e_{p q} & =R_{12 p q}, & f_{p q} & =R_{34 p q}
\end{aligned}
$$

$(5 \leq p, q \leq n)$. It follows from Proposition 8 that the matrix

$$
L=\left[\begin{array}{cccc}
B & -F & -C & -D \\
F & B & D & -C \\
-C^{T} & D^{T} & A & -E \\
-D^{T} & -C^{T} & E & A
\end{array}\right]
$$

is positive semi-definite. We next define

$$
U=\left[\begin{array}{cccc}
0 & 0 & I & 0 \\
0 & 0 & 0 & -I \\
-I & 0 & 0 & 0 \\
0 & I & 0 & 0
\end{array}\right]
$$


Since $L$ is positive semi-definite, we have

$$
\begin{aligned}
0 & \leq \frac{1}{4} \operatorname{tr}\left(L U L U^{T}\right) \\
& =\operatorname{tr}(A B)+\operatorname{tr}(E F)-\operatorname{tr}\left(C^{2}\right)-\operatorname{tr}\left(D^{2}\right) \\
& =\sum_{p, q=5}^{n} a_{p q} b_{p q}-\sum_{p, q=5}^{n} e_{p q} f_{p q}-\sum_{p, q=5}^{n} c_{p q} c_{q p}-\sum_{p, q=5}^{n} d_{p q} d_{q p} .
\end{aligned}
$$

This completes the proof.

Corollary 10. We have

$$
\begin{aligned}
& \sum_{p, q=1}^{n}\left(R_{1 p 1 q}+R_{2 p 2 q}\right)\left(R_{3 p 3 q}+R_{4 p 4 q}\right)-\sum_{p, q=1}^{n} R_{12 p q} R_{34 p q} \\
& \geq \sum_{p, q=1}^{n}\left(R_{1 p 3 q}+R_{2 p 4 q}\right)\left(R_{3 p 1 q}+R_{4 p 2 q}\right) \\
& +\sum_{p, q=1}^{n}\left(R_{1 p 4 q}-R_{2 p 3 q}\right)\left(R_{4 p 1 q}-R_{3 p 2 q}\right) .
\end{aligned}
$$

Consequently,

$$
Q(R)_{1313}+Q(R)_{1414}+Q(R)_{2323}+Q(R)_{2424}-2 Q(R)_{1234} \geq 0 .
$$

After these preparations, we now prove that nonnegative isotropic curvature is preserved by the ODE $\frac{d}{d t} R=Q(R)$ :

Proposition 11. Suppose that $R(t), t \in[0, T)$, is a solution of the $O D E \frac{d}{d t} R(t)=$ $Q(R(t))$. If $R(0)$ has nonnegative isotropic curvature, then $R(t)$ has nonnegative isotropic curvature for all $t \in[0, T)$.

Proof. Fix $\varepsilon>0$, and denote by $R_{\varepsilon}(t)$ the solution of the ODE $\frac{d}{d t} R_{\varepsilon}(t)=Q\left(R_{\varepsilon}(t)\right)+$ $\varepsilon I$ with initial condition $R_{\varepsilon}(0)=R(0)+\varepsilon I$. The function $R_{\varepsilon}(t)$ is defined on some time interval $\left[0, T_{\varepsilon}\right)$. We claim that $R_{\varepsilon}(t)$ has positive isotropic for all $t \in\left[0, T_{\varepsilon}\right)$. To prove this, we argue by contradiction. Suppose that there exists a time $t \in\left[0, T_{\varepsilon}\right)$ such that $R_{\varepsilon}(t)$ does not have positive isotropic curvature. Let

$$
\tau=\inf \left\{t \in\left[0, T_{\varepsilon}\right): R_{\varepsilon}(t) \text { does not have positive isotropic curvature }\right\} .
$$

Clearly, $\tau>0$. Moreover, there exists an orthonormal four-frame $\left\{e_{1}, e_{2}, e_{3}, e_{4}\right\}$ such that

$$
R_{\varepsilon}(\tau)_{1313}+R_{\varepsilon}(\tau)_{1414}+R_{\varepsilon}(\tau)_{2323}+R_{\varepsilon}(\tau)_{2424}-2 R_{\varepsilon}(\tau)_{1234}=0 .
$$

By definition of $\tau, R_{\varepsilon}(t)$ has positive isotropic curvature for all $t \in[0, \tau)$. This implies

$$
R_{\varepsilon}(\tau)_{1313}+R_{\varepsilon}(\tau)_{1414}+R_{\varepsilon}(\tau)_{2323}+R_{\varepsilon}(\tau)_{2424}-2 R_{\varepsilon}(\tau)_{1234}>0
$$

for all $t \in[0, \tau)$. Therefore, we obtain

$$
\begin{aligned}
& Q\left(R_{\varepsilon}(\tau)\right)_{1313}+Q\left(R_{\varepsilon}(\tau)\right)_{1414} \\
& +Q\left(R_{\varepsilon}(\tau)\right)_{2323}+Q\left(R_{\varepsilon}(\tau)\right)_{2424}-2 Q\left(R_{\varepsilon}(\tau)\right)_{1234}+4 \varepsilon \leq 0
\end{aligned}
$$


On the other hand, since $R_{\varepsilon}(\tau)$ has nonnegative isotropic curvature, we have

$$
\begin{aligned}
& Q\left(R_{\varepsilon}(\tau)\right)_{1313}+Q\left(R_{\varepsilon}(\tau)\right)_{1414} \\
& +Q\left(R_{\varepsilon}(\tau)\right)_{2323}+Q\left(R_{\varepsilon}(\tau)\right)_{2424}-2 Q\left(R_{\varepsilon}(\tau)\right)_{1234} \geq 0
\end{aligned}
$$

by Corollary 10. This is a contradiction.

Therefore, $R_{\varepsilon}(t)$ has positive isotropic curvature for all $t \in\left[0, T_{\varepsilon}\right)$. Standard ODE theory implies that $T \leq \liminf _{\varepsilon \rightarrow 0} T_{\varepsilon}$ and $R(t)=\lim _{\varepsilon \rightarrow 0} R_{\varepsilon}(t)$ for all $t \in$ $[0, T)$. Consequently, $R(t)$ has nonnegative isotropic curvature for all $t \in[0, T)$.

\section{Another invariant CURVAture CONDition fOR the Ricci Flow}

In this section, we construct a continuous family of cones that serves as a pinching family. Given any algebraic curvature operator $R$ on $\mathbb{R}^{n}$, we define an algebraic curvature operator $\hat{R}$ on $\mathbb{R}^{n} \times \mathbb{R}^{2}$ by

$$
\hat{R}\left(\hat{v}_{1}, \hat{v}_{2}, \hat{v}_{3}, \hat{v}_{4}\right)=R\left(v_{1}, v_{2}, v_{3}, v_{4}\right)
$$

for all vectors $\hat{v}_{j}=\left(v_{j}, x_{j}\right) \in \mathbb{R}^{n} \times \mathbb{R}^{2}$. We denote by $\hat{C}$ the set of all algebraic curvature operators on $\mathbb{R}^{n}$ with the property that $\hat{R}$ has nonnegative isotropic curvature:

$$
\hat{C}=\left\{R \in S_{B}^{2}(\mathfrak{s o}(n)): \hat{R} \text { has nonnegative isotropic curvature }\right\} .
$$

Clearly, $\hat{C}$ is a closed, convex, $O(n)$-invariant cone in the space of algebraic curvature operators. We next establish some basic properties of the cone $\hat{C}$ :

Proposition 12. The cone $\hat{C}$ has the following properties:

(i) The cone $\hat{C}$ is invariant under the $O D E \frac{d}{d t} R=Q(R)$.

(ii) Every algebraic curvature operator $R \in \hat{C}$ has nonnegative sectional curvature.

(iii) If $R$ is a nonnegative curvature operator on $\mathbb{R}^{n}$, then $R$ lies in $\hat{C}$.

Proof. Suppose that $R(t), t \in[0, T)$, is a solution of the ODE $\frac{d}{d t} R(t)=Q(R(t))$ with $R(0) \in \hat{C}$. Then $\hat{R}(t), t \in[0, T)$, is a solution of the analogous ODE on $\mathbb{R}^{n} \times \mathbb{R}^{2}$. Since $\hat{R}(0)$ has nonnegative isotropic curvature, Proposition 11 implies that $\hat{R}(t)$ has nonnegative isotropic curvature for all $t \in[0, T)$. Thus, we conclude that $R(t) \in \hat{C}$ for all $t \in[0, T)$.

In order to prove (ii), we consider an algebraic curvature operator $R \in \hat{C}$. Let $\left\{e_{1}, e_{2}\right\}$ be an orthonormal two-frame in $\mathbb{R}^{n}$. We define an orthonormal four-frame $\left\{\hat{e}_{1}, \hat{e}_{2}, \hat{e}_{3}, \hat{e}_{4}\right\}$ in $\mathbb{R}^{n} \times \mathbb{R}^{2}$ by

$$
\begin{array}{ll}
\hat{e}_{1}=\left(e_{1}, 0,0\right), & \hat{e}_{2}=(0,0,1), \\
\hat{e}_{3}=\left(e_{2}, 0,0\right), & \hat{e}_{4}=(0,1,0) .
\end{array}
$$

Since $\hat{R}$ has nonnegative isotropic curvature, we have

$$
\begin{aligned}
0 & \leq \hat{R}\left(\hat{e}_{1}, \hat{e}_{3}, \hat{e}_{1}, \hat{e}_{3}\right)+\hat{R}\left(\hat{e}_{1}, \hat{e}_{4}, \hat{e}_{1}, \hat{e}_{4}\right) \\
& +\hat{R}\left(\hat{e}_{2}, \hat{e}_{3}, \hat{e}_{2}, \hat{e}_{3}\right)+\hat{R}\left(\hat{e}_{2}, \hat{e}_{4}, \hat{e}_{2}, \hat{e}_{4}\right)-2 \hat{R}\left(\hat{e}_{1}, \hat{e}_{2}, \hat{e}_{3}, \hat{e}_{4}\right) \\
& =R\left(e_{1}, e_{2}, e_{1}, e_{2}\right) .
\end{aligned}
$$

Hence, $R$ has nonnegative sectional curvature. 
It remains to verify (iii). Let $R$ be a nonnegative curvature operator on $\mathbb{R}^{n}$. Let $\left\{\hat{e}_{1}, \hat{e}_{2}, \hat{e}_{3}, \hat{e}_{4}\right\}$ be an orthonormal four-frame in $\mathbb{R}^{n} \times \mathbb{R}^{2}$. We write $\hat{e}_{j}=\left(v_{j}, x_{j}\right)$, where $v_{j} \in \mathbb{R}^{n}$ and $x_{j} \in \mathbb{R}^{2}$. Letting

$$
\begin{aligned}
& \varphi=v_{1} \wedge v_{3}+v_{4} \wedge v_{2}, \\
& \psi=v_{1} \wedge v_{4}+v_{2} \wedge v_{3},
\end{aligned}
$$

we obtain

$$
\begin{aligned}
& \hat{R}\left(\hat{e}_{1}, \hat{e}_{3}, \hat{e}_{1}, \hat{e}_{3}\right)+\hat{R}\left(\hat{e}_{1}, \hat{e}_{4}, \hat{e}_{1}, \hat{e}_{4}\right) \\
& +\hat{R}\left(\hat{e}_{2}, \hat{e}_{3}, \hat{e}_{2}, \hat{e}_{3}\right)+\hat{R}\left(\hat{e}_{2}, \hat{e}_{4}, \hat{e}_{2}, \hat{e}_{4}\right)-2 \hat{R}\left(\hat{e}_{1}, \hat{e}_{2}, \hat{e}_{3}, \hat{e}_{4}\right) \\
& =R(\varphi, \varphi)+R(\psi, \psi) \geq 0 .
\end{aligned}
$$

Thus, we conclude that $R \in \hat{C}$.

We next apply a technique discovered by C. Böhm and B. Wilking [3]. For each pair of real numbers $a, b$, Böhm and Wilking define a linear transformation $\ell_{a, b}$ on the space of algebraic curvature operators by

$$
\ell_{a, b}(R)=R+b \operatorname{Ric}_{0} \oplus \mathrm{id}+\frac{a}{n} \text { scal id } \otimes \text { id. }
$$

Here, scal and Ric denote the scalar curvature and trace-free Ricci tensor of $R$, respectively. Moreover, $₫$ denotes the Kulkarni-Nomizu product, i.e.

$$
(A \oplus B)_{i j k l}=A_{i k} B_{j l}-A_{i l} B_{j k}-A_{j k} B_{i l}+A_{j l} B_{i k} .
$$

For abbreviation, let $I=\frac{1}{2}$ id $₫$ id. Combining Proposition 12 with results of Böhm and Wilking [3] yields:

Proposition 13. Let $0<b \leq \frac{1}{2}$. We define a cone $\hat{C}(b)$ by

$$
\hat{C}(b)=\left\{\ell_{a, b}(R): R \in \hat{C} \text { and } \operatorname{Ric} \geq \frac{p}{n} \text { scal }\right\},
$$

where

$$
2 a=\frac{2 b+(n-2) b^{2}}{1+(n-2) b^{2}}, \quad p=1-\frac{1}{1+(n-2) b^{2}} .
$$

Then the cone $\hat{C}(b)$ is transversally invariant under the $O D E \frac{d}{d t} R=Q(R)$. More precisely, for each $R \in \partial \hat{C}(b) \backslash\{0\}, Q(R)$ lies in the interior of the tangent cone to $\hat{C}(b)$ at $R$.

Proposition 14. Let $a>\frac{1}{2}$. We define a cone $\hat{C}(a)$ by

$$
\hat{C}(a)=\left\{\ell_{a, b}(R): R \in \hat{C} \text { and } \operatorname{Ric} \geq \frac{p}{n} \text { scal }\right\},
$$

where

$$
b=\frac{1}{2}, \quad p=1-\frac{4}{n-2+8 a} .
$$

Then the cone $\hat{C}(a)$ is transversally invariant under the $O D E \frac{d}{d t} R=Q(R)$. More precisely, for each $R \in \partial \hat{C}(a) \backslash\{0\}, Q(R)$ lies in the interior of the tangent cone to $\hat{C}(a)$ at $R$. 
The proofs of Proposition 13 and Proposition 14 are analogous to Lemma 3.4 and Lemma 3.5 in [3], respectively.

Proposition 13 and Proposition 14 provide a continuous family $\hat{C}(s), s>0$, of closed, convex, $O(n)$-invariant cones. It is easy to see that these cones form a pinching family in the sense of Böhm and Wilking [3]:

Proposition 15. The cones $\hat{C}(s), s>0$, have the following properties:

(i) For each $R \in \partial \hat{C}(s) \backslash\{0\}, Q(R)$ lies in the interior of the tangent cone to $\hat{C}(s)$ at $R$.

(ii) I lies in the interior of $\hat{C}(s)$.

(iii) Given any $\delta \in(0,1)$, there exists a real number $s>0$ such that every algebraic curvature operator $R \in \hat{C}(s) \backslash\{0\}$ is $\delta$-pinched.

The convergence of the normalized Ricci flow follows now from a result of Böhm and Wilking (cf. 3, Theorem 5.1) which in turn relies on work of Hamilton (cf. 12. Section 5). The proof of that result requires the construction of a suitable pinching set for the ODE. We have a slightly different construction of such a set, which we provide for the convenience of the reader.

Proposition 16. Fix a compact interval $[\alpha, \beta] \subset(0, \infty)$. Assume that $F_{0}$ is a closed set which is invariant under the $O D E \frac{d}{d t} R=Q(R)$. Moreover, suppose that

$$
F_{0} \subset\{R: R+h I \in \hat{C}(s)\}
$$

for some $s \in[\alpha, \beta]$ and some $h>0$. Then there exists a real number $\varepsilon>0$, depending only on $\alpha, \beta$, and $n$, such that the following hold:

(i) The set

$$
F_{1}=F_{0} \cap\{R: R+2 h I \in \hat{C}(s+\varepsilon)\}
$$

is invariant under the $O D E \frac{d}{d t} R=Q(R)$.

(ii) We have

$$
F_{0} \cap\{R: \operatorname{tr}(R) \leq h\} \subset F_{1} .
$$

Proof. For each $R \in \partial \hat{C}(s) \backslash\{0\}, Q(R)$ lies in the interior of the tangent cone to $\hat{C}(s)$ at $R$. Since $Q(R)$ is homogenous of degree 2 , we can find a constant $N>1$, depending only on $\alpha, \beta$, and $n$, with the following property: if $R \in \partial \hat{C}(s)$ for some $s \in[\alpha, \beta+1]$ and $\operatorname{tr}(R)>N$, then $Q(R-2 I)$ lies in the interior of the tangent cone to $\hat{C}(s)$ at $R$.

Observe that $\{R: R+I \in \hat{C}(s)\} \cap\{R: \operatorname{tr}(R) \leq N\}$ is a compact set which is contained in the interior of the set $\{R: R+2 I \in \hat{C}(s)\}$. Hence, there exists a real number $\varepsilon \in(0,1)$, depending only on $\alpha, \beta$, and $n$, such that

$$
\{R: R+I \in \hat{C}(s)\} \cap\{R: \operatorname{tr}(R) \leq N\} \subset\{R: R+2 I \in \hat{C}(s+\varepsilon)\}
$$

for all $s \in[\alpha, \beta]$.

We now consider the set

$$
F_{1}=F_{0} \cap\{R: R+2 h I \in \hat{C}(s+\varepsilon)\} .
$$

Using the inclusions

$$
F_{0} \subset\{R: R+h I \in \hat{C}(s)\}
$$

and

$$
\{R: R+h I \in \hat{C}(s)\} \cap\{R: \operatorname{tr}(R) \leq N h\} \subset\{R: R+2 h I \in \hat{C}(s+\varepsilon)\},
$$


we obtain

$$
F_{0} \cap\{R: \operatorname{tr}(R) \leq N h\} \subset F_{1} .
$$

Hence, it remains to show that the set $F_{1}$ is invariant under the ODE $\frac{d}{d t} R=Q(R)$. Let $R(t), t \in[0, T)$, be a solution of the ODE $\frac{d}{d t} R(t)=Q(R(t))$ with $R(0) \in F_{1}$. Since $F_{0}$ is invariant under the $\operatorname{ODE} \frac{d}{d t} R=Q(R)$, we have $R(t) \in F_{0}$ for all $t \in[0, T)$. We claim that $R(t)+2 h I \in \hat{C}(s+\varepsilon)$ for all $t \in[0, T)$. Suppose this is false. We then define

$$
\tau=\inf \{t \in[0, T): R(t)+2 h I \notin \hat{C}(s+\varepsilon)\} .
$$

Clearly, $R(\tau)+2 h I \in \partial \hat{C}(s+\varepsilon)$. Moreover, we have $\operatorname{tr}(R(\tau)) \geq N h$, hence $\operatorname{tr}(R(\tau)+$ $2 h I)>N h$. Consequently, $Q(R(\tau))$ lies in the interior of the tangent cone to $\hat{C}(s+\varepsilon)$ at $R(\tau)+2 h I$. This contradicts the definition of $\tau$. Thus, we conclude that $R(t) \in F_{1}$ for all $t \in[0, T)$.

Proposition 17 (Böhm and Wilking [3], Theorem 4.1). Suppose that $K$ is a compact set which is contained in the interior of $\hat{C}$. Then there exists a closed, convex, $O(n)$-invariant set $F$ with the following properties:

(i) $F$ is invariant under the $O D E \frac{d}{d t} R=Q(R)$.

(ii) For each $\delta \in(0,1)$, the set $\{R \in F: R$ is not $\delta$-pinched $\}$ is bounded.

(iii) $K$ is a subset of $F$.

Proof. By scaling, we may assume that $\operatorname{tr}(R) \leq 1$ for all $R \in K$. Since $K$ is contained in the interior of $\hat{C}$, there exists a positive real number $s_{0}$ such that $K \subset \hat{C}\left(s_{0}\right)$. We now apply Proposition 16 with $F_{0}=\hat{C}\left(s_{0}\right)$ and $h=1$. Hence, there exists a real number $s_{1}>s_{0}$ such that the set

$$
F_{1}=F_{0} \cap\left\{R: R+2 I \in \hat{C}\left(s_{1}\right)\right\}
$$

is invariant under the ODE $\frac{d}{d t} R=Q(R)$, and

$$
F_{0} \cap\{R: \operatorname{tr}(R) \leq 1\} \subset F_{1} .
$$

Proceeding inductively, we obtain an increasing sequence of real numbers $s_{j}, j \in \mathbb{N}$, and a sequence of closed, convex, $O(n)$-invariant sets $F_{j}, j \in \mathbb{N}$, with the following properties:

(a) For each $j \in \mathbb{N}$, we have $F_{j+1}=F_{j} \cap\left\{R: R+2^{j+1} I \in \hat{C}\left(s_{j+1}\right)\right\}$.

(b) For each $j \in \mathbb{N}$, we have $F_{j} \cap\left\{R: \operatorname{tr}(R) \leq 2^{j}\right\} \subset F_{j+1}$.

(c) For each $j \in \mathbb{N}$, the set $F_{j}$ is invariant under the ODE $\frac{d}{d t} R=Q(R)$.

(d) $s_{j} \rightarrow \infty$ as $j \rightarrow \infty$.

We now define $F=\bigcap_{j=1}^{\infty} F_{j}$. Clearly, $F$ is a closed, convex, $O(n)$-invariant set, which is invariant under the ODE $\frac{d}{d t} R=Q(R)$. Since $K \subset F_{0} \cap\{R: \operatorname{tr}(R) \leq 1\}$, it follows from property (b) that $K \subset F_{j}$ for all $j \in \mathbb{N}$. Hence, $K$ is a subset of $F$. Finally, property (a) implies

$$
F \subset F_{j} \subset\left\{R: R+2^{j} I \in \hat{C}\left(s_{j}\right)\right\}
$$

for all $j \in \mathbb{N}$. Since $s_{j} \rightarrow \infty$ as $j \rightarrow \infty$, the assertion follows from Proposition 15.

Having established the existence of suitable pinching sets, the convergence of the flow follows from the same arguments as in [3], [12]: 
Theorem 18. Let $\left(M, g_{0}\right)$ be a compact Riemannian manifold of dimension $n \geq 4$. Assume that the curvature tensor of $\left(M, g_{0}\right)$ lies in the interior of the cone $\hat{C}$ for all points in $M$. Then the normalized Ricci flow with initial metric $g_{0}$ exists for all time and converges to a metric of constant sectional curvature as $t \rightarrow \infty$.

\section{An algebraic Characterization of the CONE $\hat{C}$}

In this section, we provide a necessary and sufficient condition for $\hat{R}$ to have nonnegative isotropic curvature. We will need the following linear algebra result (cf. [6, Lemma 3.1). We give a short proof of this for completeness.

Lemma 19. Assume that $\varphi, \psi \in \wedge^{2} \mathbb{R}^{4}$ are two-vectors satisfying $\varphi \wedge \varphi=\psi \wedge \psi$, $\varphi \wedge \psi=0$, and $\langle\varphi, \psi\rangle=0$. Then there exists an orthonormal basis $\left\{e_{1}, e_{2}, e_{3}, e_{4}\right\}$ of $\mathbb{R}^{4}$ such that

$$
\begin{aligned}
& \varphi=a_{1} e_{1} \wedge e_{3}+a_{2} e_{4} \wedge e_{2}, \\
& \psi=b_{1} e_{1} \wedge e_{4}+b_{2} e_{2} \wedge e_{3}
\end{aligned}
$$

with $a_{1} a_{2}=b_{1} b_{2}$.

Proof. We first consider the (generic) case in which at least one of $\varphi, \psi$ is neither self-dual nor anti-self-dual. Without loss of generality, we may assume that $\varphi$ is neither self-dual nor anti-self-dual. Consider the anti-symmetric bilinear form defined on $\mathbb{R}^{4}$ by $(v, w) \mapsto\langle\varphi, v \wedge w\rangle$. A standard result in linear algebra implies that there exists a positively oriented orthonormal basis $\left\{v_{1}, v_{2}, v_{3}, v_{4}\right\}$ in which $\varphi$ has the form

$$
\varphi=a_{1} v_{1} \wedge v_{3}+a_{2} v_{4} \wedge v_{2}
$$

for suitable coefficients $a_{1}, a_{2}$. By assumption, we have $\langle\varphi, \psi\rangle=0$ and $\varphi \wedge \psi=0$. This implies

$$
a_{1}\left\langle\psi, v_{1} \wedge v_{3}\right\rangle+a_{2}\left\langle\psi, v_{4} \wedge v_{2}\right\rangle=0
$$

and

$$
a_{2}\left\langle\psi, v_{1} \wedge v_{3}\right\rangle+a_{1}\left\langle\psi, v_{4} \wedge v_{2}\right\rangle=0 .
$$

Since $\varphi$ is neither self-dual nor anti-self-dual, we have $a_{1}^{2} \neq a_{2}^{2}$. Therefore, we obtain $\left\langle\psi, v_{1} \wedge v_{3}\right\rangle=\left\langle\psi, v_{4} \wedge v_{2}\right\rangle=0$.

We now consider the two-dimensional subspaces $W, Z \subset \mathbb{R}^{4}$ where $W$ is the span of $\left\{v_{1}, v_{3}\right\}$ and $Z$ is the span of $\left\{v_{4}, v_{2}\right\}$. We take the orientations on these spaces so that the indicated bases are positively oriented. We consider the bilinear pairing $\sigma: W \times Z \rightarrow \mathbb{R}$ given by $\sigma(w, z)=\langle\psi, w \wedge z\rangle$. Linear algebra (singular value decomposition) allows us to find positively oriented orthonormal bases $\left\{e_{1}, e_{3}\right\}$ for $W$ and $\left\{e_{4}, e_{2}\right\}$ for $Z$ such that $\sigma\left(e_{1}, e_{2}\right)=0$ and $\sigma\left(e_{3}, e_{4}\right)=0$. Clearly, $\left\{e_{1}, e_{2}, e_{3}, e_{4}\right\}$ is a positively oriented orthonormal basis of $\mathbb{R}^{4}$. Since $e_{1} \wedge e_{3}=v_{1} \wedge v_{3}$ and $e_{4} \wedge e_{2}=v_{4} \wedge v_{2}$, we have

$$
\varphi=a_{1} e_{1} \wedge e_{3}+a_{2} e_{4} \wedge e_{2} .
$$

Moreover, we have

$$
\begin{aligned}
& \left\langle\psi, e_{1} \wedge e_{3}\right\rangle=\left\langle\psi, v_{1} \wedge v_{3}\right\rangle=0 \\
& \left\langle\psi, e_{4} \wedge e_{2}\right\rangle=\left\langle\psi, v_{4} \wedge v_{2}\right\rangle=0
\end{aligned}
$$


and

$$
\begin{aligned}
& \left\langle\psi, e_{1} \wedge e_{2}\right\rangle=\sigma\left(e_{1}, e_{2}\right)=0, \\
& \left\langle\psi, e_{3} \wedge e_{4}\right\rangle=\sigma\left(e_{3}, e_{4}\right)=0 .
\end{aligned}
$$

Thus, we conclude that

$$
\psi=b_{1} e_{1} \wedge e_{4}+b_{2} e_{2} \wedge e_{3}
$$

for suitable coefficients $b_{1}, b_{2}$. The condition $\varphi \wedge \varphi=\psi \wedge \psi$ then implies $a_{1} a_{2}=b_{1} b_{2}$.

We next consider the case in which each of $\varphi$ and $\psi$ is either self-dual or antiself-dual. The condition $\varphi \wedge \varphi=\psi \wedge \psi$ implies that they are either both self-dual or both anti-self-dual. Without loss of generality assume both are self-dual. Since the assertion is trivial for $\varphi=\psi=0$, we may assume that $\varphi \neq 0$. As above, we choose a positively oriented orthonormal basis $\left\{v_{1}, v_{2}, v_{3}, v_{4}\right\}$ in which $\varphi=a\left(v_{1} \wedge v_{3}+v_{4} \wedge v_{2}\right)$ for some $a \neq 0$. The condition $\langle\varphi, \psi\rangle=0$ implies $\left\langle\psi, v_{1} \wedge v_{3}+v_{4} \wedge v_{2}\right\rangle=0$. Since $\psi$ is self-dual, it follows that $\left\langle\psi, v_{1} \wedge v_{3}\right\rangle=\left\langle\psi, v_{4} \wedge v_{2}\right\rangle=0$. Therefore, we can complete the argument as above. This finishes the proof.

Lemma 20. Assume that $\varphi, \psi \in \wedge^{2} \mathbb{R}^{4}$ are two-vectors satisfying $\varphi \wedge \varphi=\psi \wedge \psi$ and $\varphi \wedge \psi=0$. Then there exists an orthonormal basis $\left\{e_{1}, e_{2}, e_{3}, e_{4}\right\}$ of $\mathbb{R}^{4}$ and real numbers $a_{1}, a_{2}, b_{1}, b_{2}, \theta$ such that $a_{1} a_{2}=b_{1} b_{2}$ and

$$
\begin{aligned}
\cos \theta \varphi+\sin \theta \psi & =a_{1} e_{1} \wedge e_{3}+a_{2} e_{4} \wedge e_{2}, \\
-\sin \theta \varphi+\cos \theta \psi & =b_{1} e_{1} \wedge e_{4}+b_{2} e_{2} \wedge e_{3} .
\end{aligned}
$$

Proof. We choose a real number $\theta$ such that

$$
\frac{1}{2} \sin (2 \theta)\left(|\varphi|^{2}-|\psi|^{2}\right)=\cos (2 \theta)\langle\varphi, \psi\rangle .
$$

We then define

$$
\begin{aligned}
& \tilde{\varphi}=\cos \theta \varphi+\sin \theta \psi, \\
& \tilde{\psi}=-\sin \theta \varphi+\cos \theta \psi .
\end{aligned}
$$

By assumption, we have $\varphi \wedge \varphi=\psi \wedge \psi$ and $\varphi \wedge \psi=0$. This implies

$$
\tilde{\varphi} \wedge \tilde{\varphi}-\tilde{\psi} \wedge \tilde{\psi}=\cos (2 \theta)(\varphi \wedge \varphi-\psi \wedge \psi)+2 \sin (2 \theta) \varphi \wedge \psi=0
$$

and

$$
\tilde{\varphi} \wedge \tilde{\psi}=-\frac{1}{2} \sin (2 \theta)(\varphi \wedge \varphi-\psi \wedge \psi)+\cos (2 \theta) \varphi \wedge \psi=0 .
$$

Moreover, we have

$$
\langle\tilde{\varphi}, \tilde{\psi}\rangle=-\frac{1}{2} \sin (2 \theta)\left(|\varphi|^{2}-|\psi|^{2}\right)+\cos (2 \theta)\langle\varphi, \psi\rangle=0
$$

by definition of $\theta$. Hence, the assertion follows from Lemma 19 ,

Proposition 21. Let $R$ be an algebraic curvature operator on $\mathbb{R}^{n}$, and let $\hat{R}$ be the induced algebraic curvature operator on $\mathbb{R}^{n} \times \mathbb{R}^{2}$. The following statements are equivalent:

(i) $\hat{R}$ has nonnegative isotropic curvature.

(ii) For all orthonormal four-frames $\left\{e_{1}, e_{2}, e_{3}, e_{4}\right\}$ and all $\lambda, \mu \in[-1,1]$, we have

$$
\begin{aligned}
& R\left(e_{1}, e_{3}, e_{1}, e_{3}\right)+\lambda^{2} R\left(e_{1}, e_{4}, e_{1}, e_{4}\right) \\
& +\mu^{2} R\left(e_{2}, e_{3}, e_{2}, e_{3}\right)+\lambda^{2} \mu^{2} R\left(e_{2}, e_{4}, e_{2}, e_{4}\right)-2 \lambda \mu R\left(e_{1}, e_{2}, e_{3}, e_{4}\right) \geq 0
\end{aligned}
$$


Proof. Assume first that $\hat{R}$ has nonnegative isotropic curvature. Let $\left\{e_{1}, e_{2}, e_{3}, e_{4}\right\}$ be an orthonormal four-frame in $\mathbb{R}^{n}$, and let $\lambda, \mu \in[-1,1]$. We define

$$
\begin{array}{ll}
\hat{e}_{1}=\left(e_{1}, 0,0\right), & \hat{e}_{2}=\left(\mu e_{2}, 0, \sqrt{1-\mu^{2}}\right), \\
\hat{e}_{3}=\left(e_{3}, 0,0\right), & \hat{e}_{4}=\left(\lambda e_{4}, \sqrt{1-\lambda^{2}}, 0\right) .
\end{array}
$$

Clearly, the vectors $\left\{\hat{e}_{1}, \hat{e}_{2}, \hat{e}_{3}, \hat{e}_{4}\right\}$ form an orthonormal four-frame in $\mathbb{R}^{n} \times \mathbb{R}^{2}$. Since $\hat{R}$ has nonnegative isotropic curvature, we have

$$
\begin{aligned}
0 & \leq \hat{R}\left(\hat{e}_{1}, \hat{e}_{3}, \hat{e}_{1}, \hat{e}_{3}\right)+\hat{R}\left(\hat{e}_{1}, \hat{e}_{4}, \hat{e}_{1}, \hat{e}_{4}\right) \\
& +\hat{R}\left(\hat{e}_{2}, \hat{e}_{3}, \hat{e}_{2}, \hat{e}_{3}\right)+\hat{R}\left(\hat{e}_{2}, \hat{e}_{4}, \hat{e}_{2}, \hat{e}_{4}\right)-2 \hat{R}\left(\hat{e}_{1}, \hat{e}_{2}, \hat{e}_{3}, \hat{e}_{4}\right) \\
& =R\left(e_{1}, e_{3}, e_{1}, e_{3}\right)+\lambda^{2} R\left(e_{1}, e_{4}, e_{1}, e_{4}\right) \\
& +\mu^{2} R\left(e_{2}, e_{3}, e_{2}, e_{3}\right)+\lambda^{2} \mu^{2} R\left(e_{2}, e_{4}, e_{2}, e_{4}\right)-2 \lambda \mu R\left(e_{1}, e_{2}, e_{3}, e_{4}\right),
\end{aligned}
$$

as claimed.

Conversely, assume that (ii) holds. We claim that $\hat{R}$ has nonnegative isotropic curvature. Let $\left\{\hat{e}_{1}, \hat{e}_{2}, \hat{e}_{3}, \hat{e}_{4}\right\}$ be an orthonormal four-frame in $\mathbb{R}^{n} \times \mathbb{R}^{2}$. We write $\hat{e}_{j}=\left(v_{j}, x_{j}\right)$, where $v_{j} \in \mathbb{R}^{n}$ and $x_{j} \in \mathbb{R}^{2}$. Let $V$ be a four-dimensional subspace of $\mathbb{R}^{n}$ containing $\left\{v_{1}, v_{2}, v_{3}, v_{4}\right\}$. We define

$$
\begin{aligned}
& \varphi=v_{1} \wedge v_{3}+v_{4} \wedge v_{2} \in \wedge^{2} V, \\
& \psi=v_{1} \wedge v_{4}+v_{2} \wedge v_{3} \in \wedge^{2} V .
\end{aligned}
$$

Clearly, $\varphi \wedge \varphi=\psi \wedge \psi$ and $\varphi \wedge \psi=0$. By Lemma 20, there exists an orthonormal basis $\left\{e_{1}, e_{2}, e_{3}, e_{4}\right\}$ of $V$ and real numbers $a_{1}, a_{2}, b_{1}, b_{2}, \theta$ such that $a_{1} a_{2}=b_{1} b_{2}$ and

$$
\begin{aligned}
& \tilde{\varphi}:=\cos \theta \varphi+\sin \theta \psi=a_{1} e_{1} \wedge e_{3}+a_{2} e_{4} \wedge e_{2}, \\
& \tilde{\psi}:=-\sin \theta \varphi+\cos \theta \psi=b_{1} e_{1} \wedge e_{4}+b_{2} e_{2} \wedge e_{3} .
\end{aligned}
$$

Using the first Bianchi identity, we obtain

$$
\begin{aligned}
R(\varphi, \varphi)+R(\psi, \psi) & =R(\tilde{\varphi}, \tilde{\varphi})+R(\tilde{\psi}, \tilde{\psi}) \\
& =a_{1}^{2} R\left(e_{1}, e_{3}, e_{1}, e_{3}\right)+b_{1}^{2} R\left(e_{1}, e_{4}, e_{1}, e_{4}\right) \\
& +b_{2}^{2} R\left(e_{2}, e_{3}, e_{2}, e_{3}\right)+a_{2}^{2} R\left(e_{2}, e_{4}, e_{2}, e_{4}\right) \\
& -2 a_{1} a_{2} R\left(e_{1}, e_{2}, e_{3}, e_{4}\right)
\end{aligned}
$$

Condition (ii) implies that the right hand side is nonnegative. From this, it follows that

$$
\begin{aligned}
& \hat{R}\left(\hat{e}_{1}, \hat{e}_{3}, \hat{e}_{1}, \hat{e}_{3}\right)+\hat{R}\left(\hat{e}_{1}, \hat{e}_{4}, \hat{e}_{1}, \hat{e}_{4}\right) \\
& +\hat{R}\left(\hat{e}_{2}, \hat{e}_{3}, \hat{e}_{2}, \hat{e}_{3}\right)+\hat{R}\left(\hat{e}_{2}, \hat{e}_{4}, \hat{e}_{2}, \hat{e}_{4}\right)-2 \hat{R}\left(\hat{e}_{1}, \hat{e}_{2}, \hat{e}_{3}, \hat{e}_{4}\right) \\
& =R(\varphi, \varphi)+R(\psi, \psi) \geq 0 .
\end{aligned}
$$

Hence, $\hat{R}$ has nonnegative isotropic curvature.

Corollary 22. Assume that all sectional curvatures of $R$ lie in the interval $[1,4]$. Then $\hat{R}$ has nonnegative isotropic curvature. 
Proof. Let $\left\{e_{1}, e_{2}, e_{3}, e_{4}\right\}$ be an orthonormal four-frame, and let $\lambda, \mu \in[-1,1]$. Since the sectional curvatures of $R$ lie in the interval [1,4], we have $\left|R\left(e_{1}, e_{2}, e_{3}, e_{4}\right)\right|$ $\leq 2$ by Berger's inequality (see e.g. [17]). Thus, we conclude that

$$
\begin{aligned}
& R\left(e_{1}, e_{3}, e_{1}, e_{3}\right)+\lambda^{2} R\left(e_{1}, e_{4}, e_{1}, e_{4}\right) \\
& +\mu^{2} R\left(e_{2}, e_{3}, e_{2}, e_{3}\right)+\lambda^{2} \mu^{2} R\left(e_{2}, e_{4}, e_{2}, e_{4}\right)-2 \lambda \mu R\left(e_{1}, e_{2}, e_{3}, e_{4}\right) \\
& \geq 1+\lambda^{2}+\mu^{2}+\lambda^{2} \mu^{2}-4|\lambda \mu| \\
& \geq 0 .
\end{aligned}
$$

Hence, the assertion follows from Proposition 21.

\section{REFERENCES}

[1] B. Andrews and H. Nguyen, Four-manifolds with 1/4-pinched flag curvatures, preprint (2007).

[2] M. Berger, Les variétés Riemanniennes 1/4-pincées, Ann. Scuola Norm. Sup. Pisa 14, 161170 (1960). MR0140054 (25:3478)

[3] C. Böhm and B. Wilking, Manifolds with positive curvature operator are space forms, Ann. of Math. 167, 1079-1097 (2008).

[4] S. Brendle and R. Schoen, Classification of manifolds with weakly 1/4-pinched curvatures, Acta Math. 200, 1-13 (2008). MR.2386107

[5] B. Chen and X. Zhu, Ricci flow with surgery on four-manifolds with positive isotropic curvature, J. Diff. Geom. 74, 177-264 (2006). MR2258799 (2007f:53077)

[6] H. Chen, Pointwise 1/4-pinched 4-manifolds, Ann. Global Anal. Geom. 9, 161-176 (1991). MR:1136125 (93b:53028)

[7] A. Fraser, Fundamental groups of manifolds with positive isotropic curvature, Ann. of Math. 158, no. 1, 345-354 (2003). MR1999925 (2004j:53050)

[8] D. Gromoll, Differenzierbare Strukturen und Metriken positiver Krümmung auf Sphären, Math. Ann. 164, 353-371 (1966). MR0196754 (33:4940)

[9] K. Grove, H. Karcher, and E. Ruh, Group actions and curvature, Invent. Math. 23, 31-48 (1974). MR0385750 (52:6609)

[10] K. Grove, H. Karcher, and E. Ruh, Jacobi fields and Finsler metrics on compact Lie groups with an application to differentiable pinching problems, Math. Ann. 211, 7-21 (1974). MR0355917 (50:8391)

[11] R. Hamilton, Three-manifolds with positive Ricci curvature, J. Diff. Geom. 17, 255-306 (1982). MR664497(84a:53050)

[12] R. Hamilton, Four-manifolds with positive curvature operator, J. Diff. Geom. 24, 153-179 (1986). MR $862046(87 \mathrm{~m}: 53055)$

[13] R. Hamilton, The formation of singularities in the Ricci flow, Surveys in Differential Geometry 2, 7-136 (1995). MR.1375255(97e:53075)

[14] R. Hamilton, Four-manifolds with positive isotropic curvature, Comm. Anal. Geom. 5, 1-92 (1997). MR1456308 (99e:53049)

[15] G. Huisken, Ricci deformation of the metric on a Riemannian manifold, J. Diff. Geom. 21, 47-62 (1985). MR806701 (86k:53059)

[16] H. Im Hof and E. Ruh, An equivariant pinching theorem, Comment. Math. Helv. 50, no. 3, 389-401 (1975). MR0385751(52:6610)

[17] H. Karcher, A short proof of Berger's curvature tensor estimates, Proc. Amer. Math. Soc. 26, 642-644 (1970). MR0270304 (42:5194)

[18] W. Klingenberg, Über Riemannsche Mannigfaltigkeiten mit positiver Krümmung, Comment. Math. Helv. 35, 47-54 (1961). MR.0139120 (25:2559)

[19] C. Margerin, Pointwise pinched manifolds are space forms, Geometric measure theory and the calculus of variations (Arcata 1984), 343-352, Proc. Sympos. Pure Math. 44, Amer. Math. Soc., Providence RI (1986). MR.840282 (87g:53063)

[20] M. Micallef and J.D. Moore, Minimal two-spheres and the topology of manifolds with positive curvature on totally isotropic two-planes, Ann. of Math. 127, 199-227 (1988). MR924677 (89e:53088) 
[21] M. Micallef and M. Wang, Metrics with nonnegative isotropic curvature, Duke Math. J. 72, no. 3, 649-672 (1993). MR1253619 (94k:53052)

[22] H. Nguyen, Invariant curvature cones and the Ricci flow, PhD thesis, Australian National University (2007).

[23] S. Nishikawa, Deformation of Riemannian metrics and manifolds with bounded curvature ratios, Geometric measure theory and the calculus of variations (Arcata 1984), 343-352, Proc. Sympos. Pure Math. 44, Amer. Math. Soc., Providence RI (1986). MR840284 (87i:58032)

[24] G. Perelman, The entropy formula for the Ricci flow and its geometric applications, arxiv:0211159.

[25] G. Perelman, Ricci flow with surgery on three-manifolds, arxiv:0303109.

[26] H.E. Rauch, A contribution to differential geometry in the large, Ann. of Math. 54, 38-55 (1951). MR0042765 (13:159b)

[27] E. Ruh, Krümmung und differenzierbare Struktur auf Sphären II, Math. Ann. 205, 113-129 (1973). MR.0370429 (51:6656)

[28] E. Ruh, Riemannian manifolds with bounded curvature ratios, J. Diff. Geom. 17, 643-653 (1982). MR683169 (84d:53043)

[29] M. Sugimoto, K. Shiohama, and H. Karcher, On the differentiable pinching problem, Math. Ann. 195, 1-16 (1971). MR0290291(44:7475)

Department of Mathematics, Stanford University, Stanford, California 94305

Department of Mathematics, Stanford University, Stanford, California 94305 